\title{
A Near-Quadratic Algorithm for Planning the Motion of a Polygon in a Polygonal Environment*
}

\author{
D. Halperin ${ }^{1 \dagger}$ and M. Sharir ${ }^{2}$ \\ ${ }^{1}$ Robotics Laboratory, Department of Computer Science, \\ Stanford University, Stanford, CA 94305, USA \\ halperin@cs.stanford.edu \\ ${ }^{2}$ School of Mathematical Sciences, Tel Aviv University, \\ Tel Aviv 69978, Israel \\ sharir@math.tau.ac.il \\ and \\ Courant Institute of Mathematical Sciences, New York University, \\ New York, NY 10012, USA
}

\begin{abstract}
We consider the problem of planning the motion of an arbitrary $k$-sided polygonal robot $B$, free to translate and rotate in a polygonal environment $V$ bounded by $n$ edges. We present an algorithm that constructs a single component of the free configuration space of $B$ in time $O\left((k n)^{2+\varepsilon}\right)$, for any $\varepsilon>0$. This algorithm, combined with some standard techniques in motion planning, yields a solution to the underlying motion-planning problem, within the same running time.
\end{abstract}

\footnotetext{
* Work on this paper by Dan Halperin has been supported by a Rothschild Postdoctoral Fellowship, by a grant from the Stanford Integrated Manufacturing Association (SIMA), by NSF/ARPA Grant IRI-9306544, and by NSF Grant CCR-9215219. Work on this paper by Micha Sharir has been supported by NSF Grants CCR-91-22103 and CCR-93-11127, by a Max-Planck Research Award, and by grants from the U.S.-Israeli Binational Science Foundation, the Israel Science Fund administered by the Israeli Academy of Sciences, and the G.I.F., the German-Israeli Foundation for Scientific Research and Development. A preliminary and extended version of the paper has appeared as: D. Halperin and M. Sharir, Near-quadratic bounds for the motion planning problem for a polygon in a polygonal environment, Proc. 34th IEEE Symp. on Foundations of Computer Science, 1993, pp. 382-391. Part of the work on the paper was carried out while Dan Halperin was at Tel Aviv University.

${ }^{\dagger}$ Current address: Department of Computer Science, Tel Aviv University, Tel Aviv 69978, Israel.
} 


\section{Introduction}

Let $B$ be an arbitrary polygonal object with $k$ sides, and let $V$ be an open planar polygonal region bounded by $n$ edges. The configuration space $\mathcal{C}$ of $B$ is a three-dimensional parametric space, each point of which represents a possible placement of $B$ by the parametrization $(x, y, \theta)$, where $(x, y)$ are the coordinates of some fixed reference point on $B$, and $\theta$ is the orientation of $B$ (the angle between some fixed ray attached to $B$ and the positive $x$-axis). We call a placement of $B$ a free placement if at this placement $B$ does not intersect the complement $V^{c}$ of $V$. The free configuration space of $B$, denoted $F P$, is the set of all free placements of $B$, and is clearly an open subset of $\mathcal{C}$.

The boundary of $F P$ consists of so-called semifree placements, where $B$ makes one or more contacts with $V^{\mathrm{c}}$ but the interior of $B$ remains disjoint from $V^{\mathrm{c}}$. We can describe $F P$ by defining in $\mathcal{C}$ a collection $\Sigma$ of contact surfaces, each being either the locus of all placements of $B$ at which some specific corner of $B$ touches some specific edge of $V$, or the locus of placements at which some side of $B$ touches some vertex of $V$. Clearly, each contact surface is a two-dimensional manifold with boundary (a "surface patch"), and, if we replace $\theta$ by $\tan (\theta / 2)$, the contact surfaces, as well as their bounding curves, are all algebraic of small (constant) maximum degree. The number of contact surfaces is clearly $O(k n)$.

If $B$ is placed at a free placement $Z$ and moves continuously from $Z$, then it remains free as long as the corresponding path traced in $\mathcal{C}$ does not hit any contact surface. Moreover, once this path crosses a contact surface, $B$ becomes nonfree. (For this we need to assume, as is customary in other treatments of this problem, the general position of $B$ and $V$; see [11] for a more precise definition of this notion.) It follows that the connected component of $F P$ that contains $Z$ is the cell that contains $Z$ in the arrangement $\mathcal{A}(\Sigma)$ of the contact surfaces. (The entire $F P$ is the union of a collection of certain cells in this arrangement.)

The first problem that arises is to obtain a sharp upper bound on the combinatorial complexity of a single connected component of $F P$, that is, of a single cell of $\mathcal{A}(\Sigma)$. The combinatorial complexity of such a cell is defined as the number of vertices, edges, and faces of $\mathcal{A}(\Sigma)$ that appear on the boundary of the cell. The problem has been studied in [11] (see also [3], [9], [10], and [15]) in the case where $B$ is convex. It was shown there that the complexity of the entire free configuration space $F P$ is $O\left(k n \lambda_{6}(k n)\right)$, where $\lambda_{q}(m)$ is the maximum length of Davenport-Schinzel sequences of order $q$ composed of $m$ symbols, and is nearly linear in $m$ for any fixed $q$ (see [14] for more details). In other words, the complexity of $F P$ is only nearly quadratic in $k n$, as opposed to a naive bound $O\left((k n)^{3}\right)$, which is a (worst-case tight) bound on the overall number of vertices in any three-dimensional arrangement of $O(k n)$ algebraic surface patches of constant maximum degree.

Unfortunately, if $B$ is not convex, the entire free configuration space of $B$ can have $\Theta\left((k n)^{3}\right)$ vertices in the worst case, as is illustrated in Fig. 1. Hence, to obtain a subcubic bound, it makes sense to focus on just a single cell of the arrangement, as we have indeed indicated above. After the original submission of this paper, the authors have shown in [8] that the complexity of a single cell in any arrangement of $N$ algebraic surface patches in $\mathbb{R}^{3}$ of constant maximum degree, bounded by algebraic arcs which also have constant maximum degree, is $O\left(N^{2+\varepsilon}\right)$, for any $\varepsilon>0$, where the constant of proportionality 


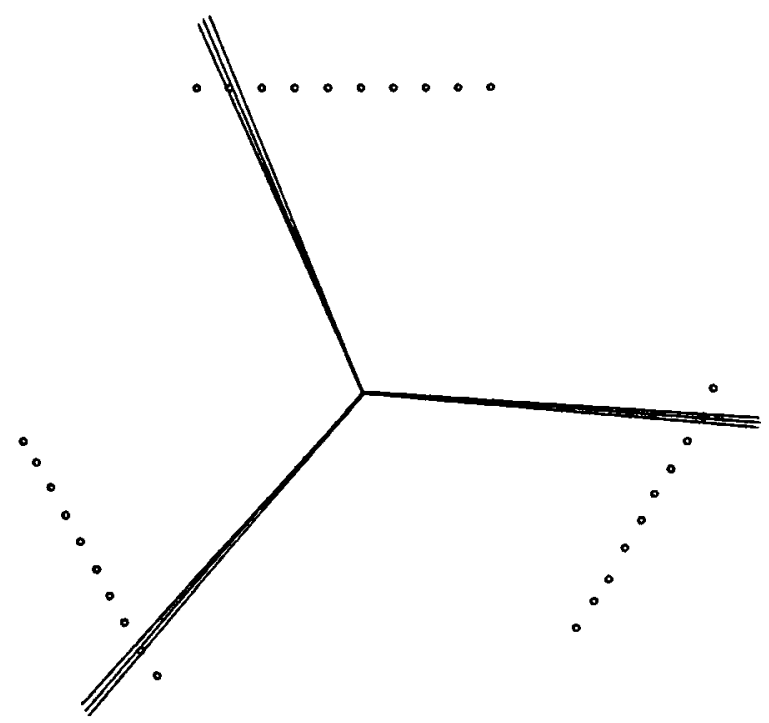

Fig. 1. An example where the entire free configuration space of a nonconvex polygon has cubic complexity.

depends on $\varepsilon$ and on the maximum degree and shape of the given surfaces and of their boundaries. Prior to this, slightly better bounds have been obtained for certain special cases, including the case of spheres, where a (worst-case tight) quadratic bound is known [13], the case of triangles, where an $O\left(N^{2} \log N\right)$ bound is known [2], and several special cases that arise in motion planning for various robot systems $B$ with three degrees of freedom, including several restricted cases of the polygon motion-planning problem that we consider here, where the shape of $B$ and/or the shape of $V$ is further restricted; these latter bounds are also all close to quadratic, and are reported in [6]. See also two recent surveys [5] and [7] for more details concerning motion-planning problems and arrangements of surfaces.

In this paper we exploit the new bounds derived in [8], introduce a special cell decomposition scheme for the cell arising in our motion-planning problem, and obtain an efficient algorithm for constructing such a cell. The algorithm runs in time $O\left((k n)^{2+\varepsilon}\right)$, for any $\varepsilon>0$, where $k$ and $n$ are as above. The new cell decomposition technique that we develop here for the algorithm may be useful for other applications as well.

We also mention that in the preliminary version of this paper, which appeared before the bounds of [8] were obtained, we showed that the complexity of a single cell in the arrangement that arises in our motion-planning problem is $k^{3} n^{2} 2^{O\left(\log ^{2 / 3} n\right)}$. When $k$ is constant, this is slightly better than the general bound of [8]. In fact, the analysis of [8] adapted and extended the technique that we used in the earlier version of this paper.

\section{Efficient Construction of a Single Cell}

In this section we obtain an efficient randomized algorithm (which can also be made deterministic) for constructing a single cell of the free configuration space of a moving 
$k$-sided polygon $B$. The general approach is similar to that of [1] and [2]. That is, let $\Sigma$ denote the collection of contact surfaces induced by the problem, and let $Z$ be a given free placement of $B$; our goal is to compute the cell containing $Z$ in $\mathcal{A}(\Sigma)$. We choose a random sample $\mathcal{R}$ of $r$ surfaces of $\Sigma$, where $r$ is some sufficiently large integer constant. We construct (e.g., by brute force) the cell $C_{0}$ containing $Z$ in $\mathcal{A}(\mathcal{R})$, and decompose $C_{0}$, in a manner to be described shortly, into subcells, each having "constant description complexity" (meaning that each cell is defined by a constant number of polynomial equalities and inequalities of constant maximum degree). The standard theory of $\varepsilon$-nets and finite VC-dimension implies that, with high probability, none of the subcells in the decomposition is crossed by more than $O((n / r) \log r)$ surfaces of $\Sigma$ (see, e.g., Appendix 7.2 of [14]). For each subcell $\xi$ we find the subcollection $\Sigma_{\xi}$ of surfaces that cross $\xi$, and compute recursively the cell $C_{\xi}$ containing $Z$ in the arrangement of these surfaces. We then form the desired cell $C$ containing $Z$ in $\mathcal{A}(\Sigma)$ by "gluing" together pieces of these cells. Specifically, we start with the subcell $\xi$ containing $Z$, and take the connected component $K$ of $\xi \cap C_{\xi}$ that contains $Z$ (note that $\xi \cap C_{\xi}$ need not be connected). If that component is disjoint from $\partial \xi$, then this is the entire desired cell $C$. Otherwise, let $f$ be a connected face of $C_{\xi} \cap \partial \xi$. We find the other subcell(s) $\xi^{\prime}$ whose boundary contains or overlaps $f$ (since our decomposition will not necessarily be a cell complex, there might be several such subcells $\xi^{\prime}$ ). We find the connected component of $\xi^{\prime} \cap C_{\xi^{\prime}}$ whose boundary contains (or overlaps) $f$, and glue that component to $K$ along $f$. We continue this gluing procedure in, say, a breadth-first style, across all subcells of $C_{0}$, until no more gluing is possible, in which case we have obtained the desired cell $C$. We refer the reader to [1] and [2] for more details. (We note that $C$ can also be constructed using the recent randomized incremental technique of [4]. Both methods, however, rely on the existence of an efficient cell decomposition scheme, like the one about to be described.)

The performance of this algorithm crucially depends on the number of subcells $\xi$ in the decomposition of $C_{0}$. We describe such a decomposition that has only $O\left(r^{2+\varepsilon}\right)$ subcells, for any $\varepsilon>0$. A standard calculation then implies that the expected running time of the algorithm is $O\left((k n)^{2+\varepsilon}\right)$, for any $\varepsilon>0$; see below for details.

The decomposition proceeds as follows. Any $\theta$-cross-section of $\mathcal{A}(\mathcal{R})$ consists of $r$ line segments. Indeed, when $\theta$ is fixed, $B$ can only translate, and the locus of all translated placements of $B$ at which it makes some specific edge-vertex contact is a line segment. Moreover, if we sweep a plane parallel to the $x y$-plane through the arrangement, the motion of the segments on the sweep plane is rather simple and has the following properties (which are easy to verify): For any pair of segments $s, s^{\prime}$, an endpoint of $s$ has the same $x$-coordinate as an endpoint of $s^{\prime}$ at most a constant number of times; and an endpoint of $s$ intersects the interior of $s^{\prime}$ at most a constant number of times.

Now, fix a segment endpoint and, at every $\theta$, extend a vertical segment (parallel to the $y$-axis) up and down from that endpoint until it hits another segment, or else extends to infinity. We consider the union of these extensions, over all values of $\theta$, as a collection of patches on an additional surface. A similar collection of patches is obtained for every other segment endpoint, so we obtain a total of at most $2 r$ additional "surfaces."

Arguing as in Lemma 6.12 of [6], it can be shown that at most $O\left(r^{2}\right)$ new faces are added to $\mathcal{A}(\mathcal{R})$ by inserting these "extension surfaces." Specifically, as long as a segment endpoint $p$ remains in the same face $f$ of the $\theta$-cross-section of $\mathcal{A}(\mathcal{R})$, the extension segment from $p$ traces a single face $\varphi$ of the corresponding extension surface. Vertices 


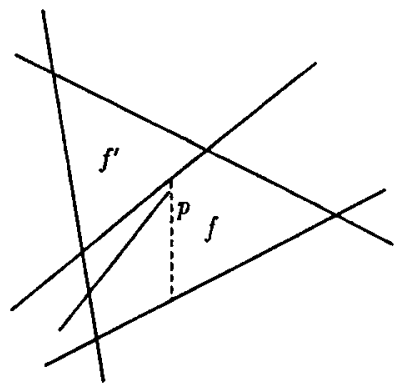

cross-section at $\theta_{0}-\varepsilon$

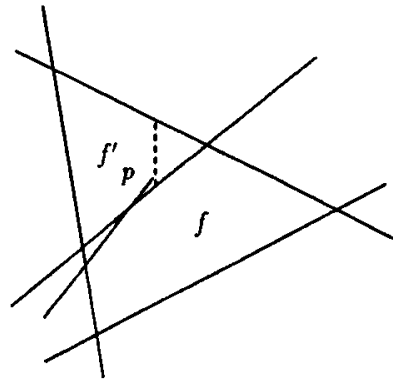

cross-section at $\theta_{0}+\varepsilon$

Fig. 2. The change in the extension segment as $p$ crosses another segment at $\theta_{0}$; both faces $f$ and $f^{\prime}$ at $\theta_{0}$ are added to the arrangement, each split into two subfaces by the extension segment.

of $\varphi$ arise when the extension segment hits a vertex of $f$, which may also be another segment endpoint vertically visible from $p$ (in the $y$-direction). Note that the collection of extension segments within a face $f$ of a $\theta$-cross-section partition it into several subfaces, and that as $\theta$ varies some of these subfaces can shrink and disappear, and be replaced by new subfaces, when pairs of extension segments overlap within $f$. Suppose that at some $\theta_{0}$ the point $p$ crosses into another face $f^{\prime}$ of the $\theta$-cross-section. Then $\varphi$ terminates at $\theta_{0}$, and a new face $\varphi^{\prime}$ begins at this orientation along the extension surface. In this case we also add to our three-dimensional arrangement $\mathcal{A}(S)$ all the "horizontal" subfaces of $f$ and of $f^{\prime}$ within the $\theta_{0}$-cross-section, which are adjacent to the respective extension segments through $p$. See Fig. 2 for an illustration of this process.

It thus follows that the overall number of faces added is $O\left(r^{2}\right)$; indeed, each crossing of a segment endpoint through another segment induces only a constant number of new faces, and the number of such crossings is $O\left(r^{2}\right)$. Note also that new subcells may start and end at $\theta$-cross-sections in which two segment endpoints become vertically visible (in the $y$-direction) within a face of the cross section (see Fig. 3 for an illustration), but the number of such events is also only $O\left(r^{2}\right)$. Consequently, a single cell is divided by these extra surfaces into at most $O\left(r^{2}\right)$ three-dimensional subcells.

As is easily verified, each of the resulting subcells $\tau$ has the property that its $\theta$-crosssection is always a convex polygon, and it varies continuously (in the Hausdorff metric of sets) with $\theta$. This is easily seen to imply that every such subcell has a unique minimum and maximum in $\theta$. As discussed in [6], the minimum need not be restricted to a single
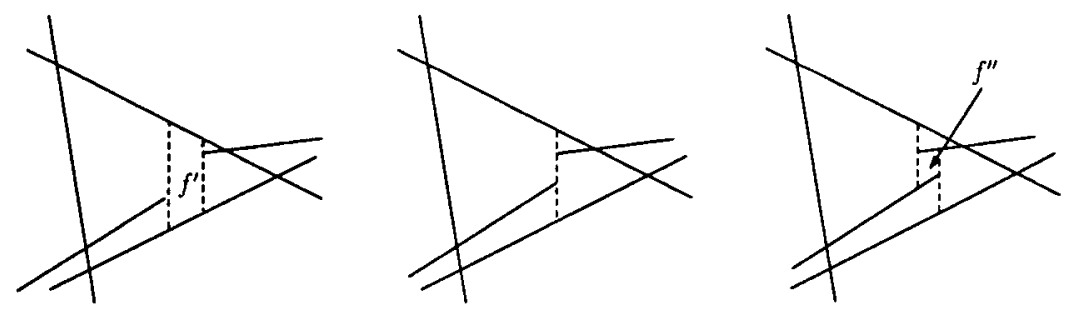

Fig. 3. A subcell whose cross section is $f^{\prime}$ terminates at $\theta_{0}$, and a new subcell whose cross section is $f^{\prime \prime}$ begins at $\theta_{0}$. 
point, and might be attained by a line segment or a two-dimensional face on the boundary of the cell, but this will not affect our analysis. There is also the special case of minima lying on the plane $\theta=0$ (containing the marking point of the cell), since we want to consider this plane as another surface in our arrangement. This way we may have added up to $O\left(r^{2}\right)$ additional local $\theta$-minima of cells.

It can also be shown that the combinatorial complexity of the faces that we have added to $\mathcal{A}(\mathcal{R})$ by the extensions from segment endpoints is $O\left(r \lambda_{6}(r)\right)$. To see this, note that a vertex of such a face $\varphi$ arises when the corresponding endpoint $p$ sees a vertex of the face $f$ containing $p$ in some $\theta$-cross-section, in the vertical $y$-direction. We can therefore define, for each of the given $N$ surfaces $\sigma$, a (partial) function $F_{\sigma}(\theta)$ which is equal to the $y$-vertical distance from $p$ to the segment $\sigma_{\theta}$, the $\theta$-cross-section of $\sigma$, whenever this distance is defined and $p$ lies, say, below that segment (in the $y$-direction). It follows that each vertex of any face $\varphi$ associated with $p$ corresponds to a breakpoint in the lower envelope of the functions $F_{\sigma}(\theta)$, or in the upper envelope of a symmetric collection of functions, each defined when $p$ lies above the corresponding segment $\sigma_{\theta}$. Using the analysis of [11], it can be shown that any pair of these functions intersect in at most four points, so the number of breakpoints of the envelopes defined for each endpoint $p$ is $O\left(\lambda_{6}(r)\right.$ ) (see [14]), from which the claim follows easily.

In contrast, we do not have equally sharp bounds for the complexity of the "horizontal" faces that are also added to $\mathcal{A}(\mathcal{R})$ in the above analysis. We suspect that their overall complexity is also roughly quadratic in $r$, but so far we were not able to show this. Our decomposition scheme will finesse this issue.

To recap, we have decomposed $C_{0}$ into $O\left(r^{2}\right)$ subcells, each of which has the property that all its $\theta$-cross-sections are convex and vary continuously with $\theta$. Moreover, it follows from the above analysis, and from the general bound of [8] on the complexity of (the undecomposed) $C_{0}$, that the total combinatorial complexity of all these subcells, excluding the complexity of the horizontal faces added in the decomposition, is $O\left(r^{2+\varepsilon}\right)$, for any $\varepsilon>0$.

We next further decompose each of these subcells as follows. Imagine that we sweep (the decomposed) $C_{0}$ with a plane $P$ parallel to the $x y$-plane, in the direction of increasing $\theta$. Let $C_{0}(\theta)$ denote the cross section $P \cap C_{0}$ when $P$ is at height $\theta$. We maintain a balanced triangulation of each convex face $f$ of $C_{0}(\theta)$ and update it whenever $P$ sweeps over a vertex of $f$, or when faces of $C_{0}(\theta)$ disappear, newly appear, split, or merge. The triangulations are balanced in the sense that the dual graph of each triangulation is a balanced binary tree whose depth is thus only logarithmic. For specificity, we use red-black trees, as described in Chapter 4 of [17]. (As defined, this dual tree is unrooted, but we root it at some arbitrary triangle incident to at least one edge of $f$.) In particular, no vertex of any face $f$ is incident to more than $O(\log r)$ triangles, and the intersection of any line with a face $f$ meets no more than $O(\log r)$ triangles (in both cases, the triangles form a path in the dual tree). For a discussion on the relation between triangulations and binary trees, see, e.g., [16]. See also Fig. 4 for an illustration of a tree corresponding to a balanced triangulation. Note that a balanced binary tree with $n$ nodes corresponds to a triangulation of a convex polygon with $n+2$ vertices, having therefore $n$ triangles. We choose an appropriate edge of the polygon to be the root edge (so that the resulting rooted tree is balanced), and label all the vertices not incident to the root edge, with an increasing sequence of integers in counterclockwise order. The triangle incident to the 

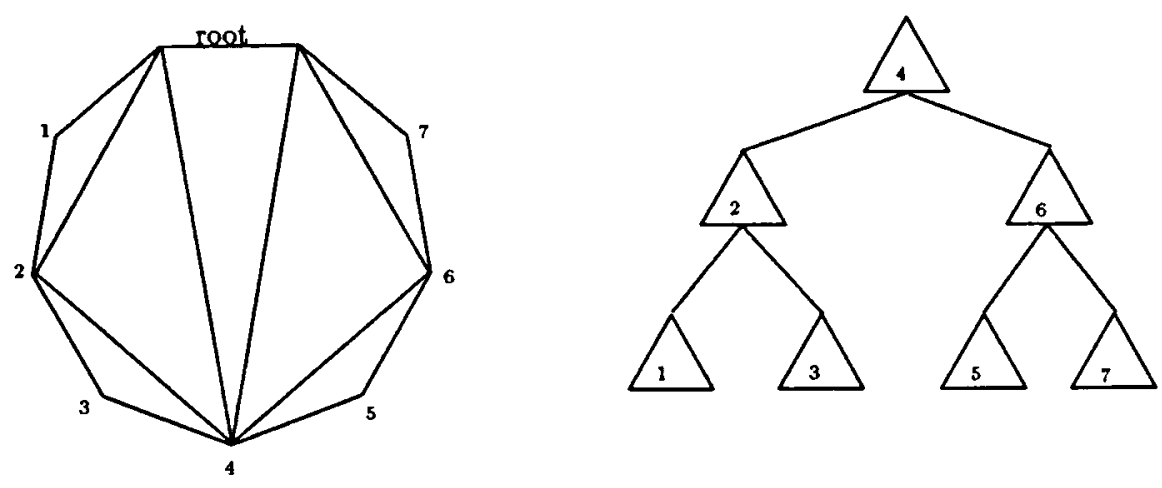

Fig. 4. A balanced triangulation of a convex polygon and the corresponding balanced binary tree; each triangle is labeled by the vertex opposite to the side it shares with its parent.

root edge corresponds to the root of the tree, and the key attached to the root of the tree is the number of the vertex of the root triangle not incident to the root edge. Each of the two nonroot edges of the root triangle may have a child triangle incident to that edge; its key is the number of the vertex of that triangle not incident to the parent (root) triangle. The labeling of tree nodes continues recursively in this manner; see Fig. 4.

(Before proceeding, it is instructive to note that the need to maintain balanced triangulations is forced on us by the fact that we do not have a near-quadratic bound on the overall complexity of the horizontal faces added in the first decomposition step. If we had such a bound, we could have afforded to use any triangulation of the faces of the cross section, because the overall number of triangles would have also been near-quadratic. We still need to triangulate these faces, to ensure that we get subcells with constant description complexity.)

For each triangle $\Delta$ we compute two critical orientations $\theta_{1}<\theta_{2}$ at which $\Delta$ is respectively "opened" (newly added to the triangulation) and "closed" (removed from the triangulation). At any time during the sweep, we store with each triangle in the current triangulation the critical orientation $\theta_{1}$ at which it was opened. Such a triangle $\Delta$ induces a subcell

$$
\xi_{\Delta}=\left\{(x, y, \theta) \mid \theta_{1} \leq \theta \leq \theta_{2},(x, y) \in \Delta(\theta)\right\},
$$

where $\Delta(\theta)$ is the set of points occupied by $\Delta$ at the cross section $C_{0}(\theta)$. It is clear that $\xi_{\Delta}$ has constant description complexity, ${ }^{1}$ and that the collection of these subcells forms a decomposition of $C_{0}$ (which is a refinement of the first decomposition, obtained above). The main goal of the following analysis is to estimate the number of subcells $\xi_{\Delta}$ that are created by the sweeping process. As we will see, the fact that the triangulations that we maintain are all balanced is crucial for the analysis. We also note that, even though the subcell decomposition is described below algorithmically, we are only concerned with its output size (namely, with the number of triangles being created), and not with its running time, since we are dealing with a constant-size problem.

\footnotetext{
${ }^{1}$ Strictly speaking, since this property requires that the subcell be represented by polynomial equalities and inequalities, we should have replaced the third coordinate $\theta$ by $\tan (\theta / 2)$; however, for convenience of presentation, we continue to denote this coordinate by $\theta$.
} 
A new face of $C_{0}(\theta)$ is formed either when a connected component of $C_{0}(\theta)$ newly appears, or when a pair of adjacent convex faces of $C_{0}(\theta)$ merge into a new (convex) face (when a $y$-vertical segment separating them is removed), or when a face of $C_{0}(\theta)$ is split into two subfaces (when a $y$-vertical segment separating them is added). When a new component of $C_{0}(\theta)$ appears, as $\theta$ slightly increases, the component becomes a triangle; thus initializing the triangulation for a new component is trivial. To initialize the whole structure at $\theta=0$, we simply triangulate each convex face of (the vertically decomposed) $C_{0}(0)$ in a balanced manner, and open all the resulting triangles at $\theta=0$.

When the sweep plane $P$ reaches a vertex $u$ of some subcell $\tau$ of $C_{0}$ at an orientation $\theta_{u}$ (excluding vertices that lie on an added horizontal face which delimits $\tau$ from above or from below), one of several types of combinatorial changes can occur at $u$ : an edge of the $\theta$-cross-section $\tau(\theta)$ may shrink to a point and disappear, or a new edge of $\tau(\theta)$ may appear, or, when we encounter at $\theta_{u}$ an edge of $\tau$ parallel to the $x y$-plane, an edge of $\tau(\theta)$ may be replaced by another edge, or the entire face $\tau(\theta)$ may shrink to a long and thin trapezoid which is finally "squashed" at $\theta_{u}$. Nevertheless, such a change at $u$ affects only a constant number of edges and vertices of $\tau(\theta)$, and thus affects only $O(\log r)$ triangles in the current triangulation of $\tau(\theta)$. Each of these triangles is closed at $\theta_{u}$ (so the subcells corresponding to these triangles are now fully defined), and $O(\log r)$ new triangles are formed as appropriate, replacing the old affected triangles; the new triangles are opened at $\theta_{u}$.

Of course, after each such update we need to rebalance the dual tree of the triangulation of $\tau(\theta)$, if necessary. For this, we can use any of the known techniques for maintaining balanced binary trees; as already mentioned, we use, for specificity, the red-black tree technique, as described in Chapter 4 of [17]. We observe that, since the triangulation that we maintain is of a convex polygon, any rotation that we want to apply to the dual tree, as an abstract structure, can be achieved by a straightforward retriangulation, in which the few triangles whose corresponding nodes have to be rotated in the tree are replaced by a few other triangles that represent these nodes after the rotation. Specifically, a single rotation in the binary tree corresponds to an edge flip in the triangulation; that is, for a pair of triangles sharing a diagonal in the triangulation, an edge flip is carried out by removing that diagonal-temporarily obtaining a (convex) quadrangle-and inserting the other diagonal of that quadrangle. Figure 5 shows how single and double rotations are implemented by edge flips. We denote the balanced tree corresponding to the triangulated polygon $f$ by $T(f)$.

We begin by describing in detail the operations that need to be performed when removing a vertex from the boundary of a convex face $f$. Adding a vertex to $f$ can be done in a similar fashion. (The actual updating of $f$, occurring when an edge of $f$ shrinks and disappears, or newly appears, can be implemented by removing two or one vertices from $f$, and then adding one or two new vertices to $f$, respectively.) We then describe how to update the triangulations when faces are merged or split.

Let $u$ be the vertex of $f$ that we are about to remove. By the above discussion, there are at most $O(\log r)$ triangles of the triangulation of $f$ that are incident to $u$, and the nodes corresponding to these triangles in the tree form a path $\pi$ of the tree. As is easily seen, the triangles incident to $u$ form a convex polygon, all of whose vertices are vertices of $f$. If the path $\pi$ contains the root node, then we denote this polygon by $g$. Otherwise, we 

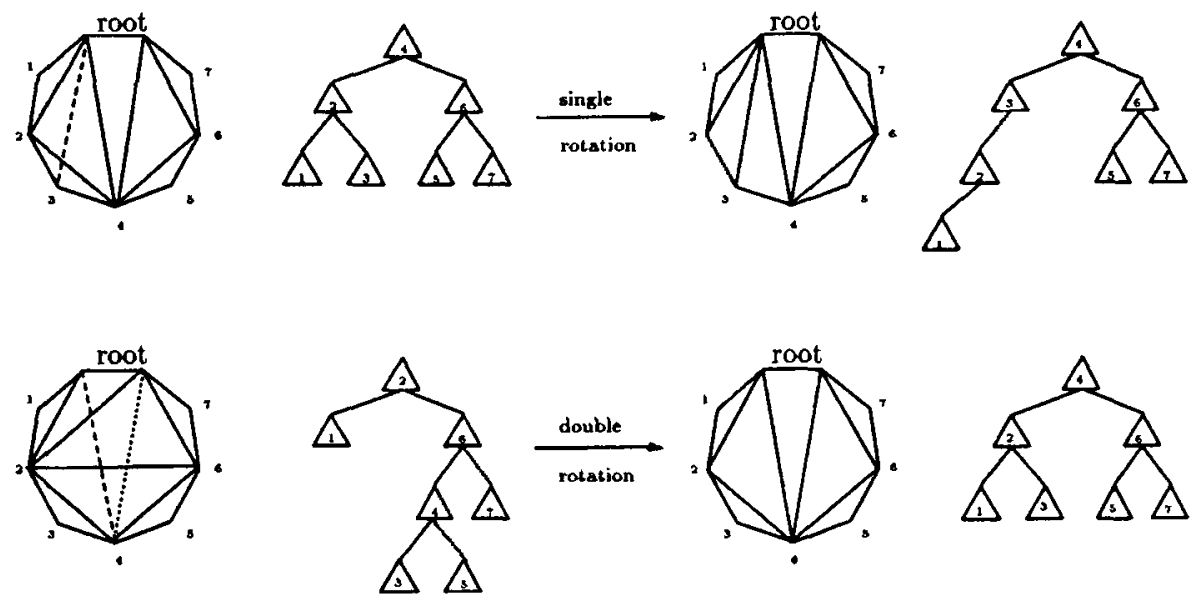

, first flip; ........, second flip.

Fig. 5. Realization of a rotation by retriangulation of a face.

follow a path $\pi^{\prime}$ from the root until it hits a node of $\pi$, add the triangles corresponding to the nodes of $\pi^{\prime}$ to those incident to $u$, and let $g$ denote the convex polygon covered by all these triangles; again, the number of original triangles contained in $g$ is at most $O(\log r)$. Let $h_{1}, h_{2}, \ldots, h_{m}$ be the $O(\log r)$ connected components of $f \backslash g$. See Fig. 6(a,b) for an illustration (the asterisks in Fig. 6(a) denote the triangles incident to $u$ ). In the example depicted in Fig. 6 , there is only one node on the path $\pi^{\prime}$, namely the root node, which is thus added to the triangles incident to $u$ in order to form $g$. Clearly, each of the $h_{i}$ 's is represented by a subtree of $T(f)$, and hence their corresponding subtrees $T\left(h_{i}\right)$ are all balanced.

We now remove the vertex $u$ from the boundary of $f$ and of $g$ to obtain a new face $f^{\prime}$ and a new portion $g^{\prime}$ thereof, respectively. Note that the polygons $h_{1}, h_{2}, \ldots, h_{m}$ are unaffected by this removal, and only $g$ changes (into $g^{\prime}$ ). Next, we retriangulate $g^{\prime}$ into $O(\log r)$ triangles such that each diagonal in this triangulation is incident to one of the endpoints of the root edge to obtain $T\left(g^{\prime}\right)$; see Fig. 6(c). For each triangle in $T\left(g^{\prime}\right)$ that shares with some $h_{i}$ an edge not incident to the root edge, we add that triangle to $h_{i}$ (making it the new root of $T\left(h_{i}\right)$ ), and remove it from the collection $T\left(g^{\prime}\right)$ (thus the remaining triangles no longer form a triangulation of $g^{\prime}$ ). It is easily verified that this process has split the face $f^{\prime}$ into subfaces $f_{1}, \ldots, f_{q}$, such that the numbers attached to the vertices of each subface form a contiguous interval of the numbers in $f^{\prime}$, and that these intervals have pairwise disjoint interiors. We number these faces in increasing order of the corresponding intervals, which corresponds to their counterclockwise order around $f^{\prime}$; see Fig. 6(d) for an illustration.

We now proceed to perform a series of join operations, as in Chapter 4 of [17], taking care that when we join two polygons $p_{1}$ and $p_{2}$, the numbers attached to vertices of $p_{1}$ are all smaller than those attached to vertices of $p_{2}$. Thus, we join $T\left(f_{1}\right)$ to $T\left(f_{2}\right)$, then we join the resulting tree to $T\left(f_{3}\right)$, and so on, until all the trees $T\left(f_{i}\right)$ have been joined. This way we obtain a balanced triangulation $T\left(f^{\prime}\right)$ of the updated face $f^{\prime}$. The cost (i.e., the number of tree operations) of each join operation is $O(\log r)$, and we repeat it 


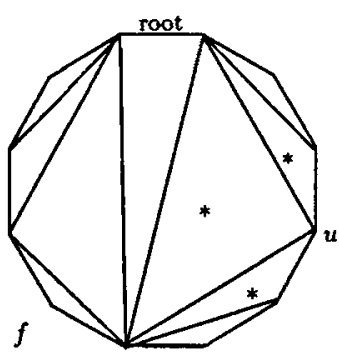

(a)

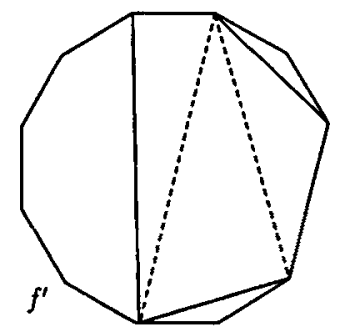

(c)

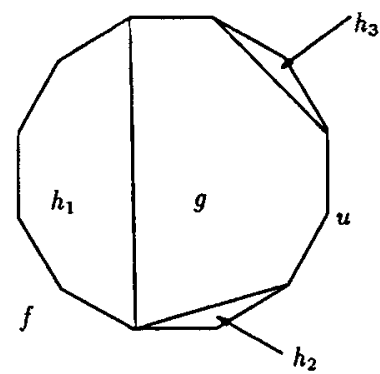

(b)

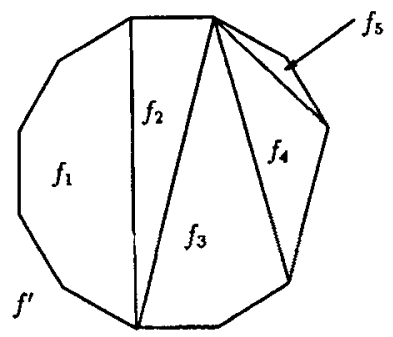

(d)

Fig. 6. Removing a vertex from a convex face.

$O(\log r)$ times. Thus the overall cost of this deletion is $O\left(\log ^{2} r\right)$; in other words, only $O\left(\log ^{2} r\right)$ new triangles are created.

The addition of a new vertex to a face $f$ is performed in a similar manner; it is somewhat simpler, because it calls for adding just one new triangle to $f$.

Remarks. (1) The join operation join $\left(s_{1}, i, s_{2}\right)$ described in Chapter 4 of [17] is defined for two trees $s_{1}$ and $s_{2}$, and for an additional element $i$, such that all the keys in $s_{1}$ are smaller than $\operatorname{key}(i)$ and all the keys in $s_{2}$ are greater than $\operatorname{key}(i)$. To conform to this notation, we note that the diagonal shared by the two subfaces that we join can play the role of the element $i$, by "thickening" it into a very thin triangle.

(2) An additional technical issue is that join $\left(s_{1}, i, s_{2}\right)$ proceeds by "hanging" $i$ as the right child of some node $w$ lying on the rightmost path of $s_{1}$, and by making the original right child $w^{\prime}$ of $w$ (if any) the left child of $i$. To implement this step via a retriangulation, let $w_{1}=w^{\prime}, w_{2}, \ldots, w_{t}$ be the nodes on the rightmost path of $s_{1}$ from' $w^{\prime}$ downward. As is easily seen, these nodes correspond to triangles, all of which are incident to a common vertex $u$ and arranged around $u$ in counterclockwise order. Let $v_{1}, v_{2}, \ldots, v_{t+1}$ be the other vertices of these triangles, arranged in counterclockwise order around the face, and let $v_{t+2}$ be the other vertex of (the "thickened") $i$; see Fig. 7. We now perform one "giant" edge flip: connect $v_{1}$ with $v_{t+2}$ by a diagonal, let $i$ now denote the new triangle spanned by $u$ and by this diagonal, and form new triangles representing $w_{1}, \ldots, w_{t}$ by connecting $v_{t+2}$ with $v_{1} v_{2}, \ldots, v_{t} v_{t+1}$, respectively; see Fig. 7 for an illustration. The remaining steps of the join are easy to implement by standard edge flips, as above. 

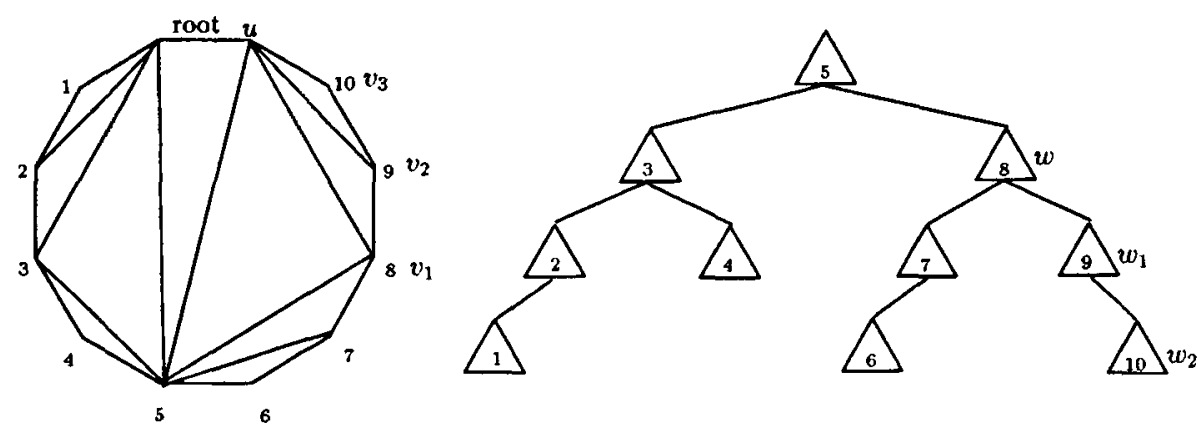

before
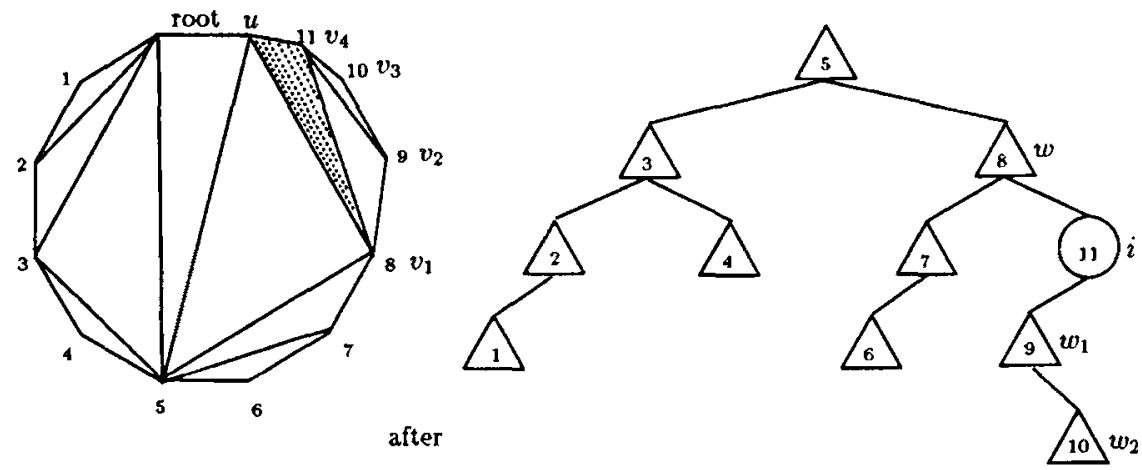

Fig. 7. The retriangulation corresponding to the hanging of a node $i$ on a node $w$ in the rightmost path of a tree.

Things are not much different when the sweep reaches an event at which two faces of $C_{0}(\theta)$ merge into a new face, or a face is split into two subfaces. Consider first the latter situation, let $f$ denote the face before splitting, and let $f_{1}$ and $f_{2}$ denote the subfaces formed by the split. A naive way of handling this configuration is to close all triangles in the current triangulation of $f$, compute balanced triangulations of $f_{1}$ and $f_{2}$ from scratch, and open all these triangles. However, the cost of this approach would be proportional to the complexity of $f$, and, as noted above, we do not have a near-quadratic bound on the overall complexity of all such faces $f$. We thus use the following more refined procedure. Let $e$ denote the $y$-vertical segment that splits $f$. Since $e$ cuts the boundary of any triangle in at most two points, it easily follows, as noted above, that the set of triangles in the triangulation of $f$ which are crossed by $e$ form a single path $\pi$ in the dual (unrooted) tree of the triangulation, or at most two paths in its rooted version $T(f)$. Thus the number of such triangles is only $O(\log r)$. Let $g$ denote the polygon covered by these triangles. As before, let $h_{1}, \ldots, h_{m}$ denote the connected components of $f \backslash g$, each of which is clearly a convex region. Some of the $h_{i}$ 's are contained in $f_{1}$ while the others are contained in $f_{2}$. We split $g$ into two subfaces $g_{1}$ and $g_{2}$, using the $y$-vertical edge $e$. $^{2}$ The edge $e$ splits the edges of $f$ into two chains, one of which contains the root edge, say the boundary of $f_{1}$. In this case we extend $g_{1}$ as before until it contains the

\footnotetext{
${ }^{2}$ Note that the endpoints of $e$ need not necessarily be vertices of $f$. To be precise, we first have to add the endpoints of $e$ as new vertices of $f$, and then proceed with the splitting operation as described above.
} 
root edge, and update the $h_{i}$ 's accordingly. Note that in $f_{1}$ the root edge is the same root edge as in $f$, and in $f_{2}$ the newly added $y$-vertical edge $e$ is the root edge. From this point, we proceed to handle each subface $f_{1}, f_{2}$ as in the case of deleting a vertex. A similar, though slightly different, procedure is used if the root edge is split by $e$.

Next consider a merge of two faces $f_{1}, f_{2}$, into a common convex face $f$, caused by removing a $y$-vertical edge $e$ separating between $f_{1}$ and $f_{2}$. Let $\pi_{1}$ (resp. $\pi_{2}$ ) denote the path in $T\left(f_{1}\right)$ (resp. in $T\left(f_{2}\right)$ ) from the root to the triangle incident to $e$ in $f_{1}$ (resp. in $f_{2}$ ). The union of all $O(\log r)$ triangles in these two paths is a convex polygon $g$ contained in $f$. As above, let $h_{1}, \ldots, h_{m}$ denote the connected components of $f \backslash g$, each of which is a convex polygon. Let $r_{1}$ be the root edge of $T\left(f_{1}\right)$. We renumber the vertices of $f$ so that they form an increasing sequence of integers in their counterclockwise order along $f$, going from $r_{1}$ all the way around. (This is an expensive operation, but, as noted above, we are only concerned with the number of triangles that the algorithm produces, and not with its running time.) We now retriangulate $g$ and perform a series of join operations, exactly as in the case, described above, of deleting a vertex, where the joins are performed according to the new order of the $h_{i}$ 's.

The final type of update occurs when a connected component of $C_{0}(\theta)$ shrinks to a point and disappears. In this case we simply close the single triangle of its current triangulation, and remove its singleton dual tree from our forest. This completes the description of our triangulation of the cell $C_{0}$.

Note that each vertex of $C_{0}$ (excluding those on the added horizontal faces) causes the generation of only $O\left(\log ^{2} r\right)$ subcells, and the same holds for each added horizontal face (whose number is only $O\left(r^{2}\right)$ ). It follows that the number of subcells in the decomposition of $C_{0}$ is $O\left(r^{2+\varepsilon}\right) \cdot O\left(\log ^{2} r\right)=O\left(r^{2+\varepsilon}\right)$, for any $\varepsilon>0$ (with a slightly larger $\varepsilon$ in the second exponent), as claimed.

The analysis of the expected running time of the algorithm is now straightforward, and similar to that in [1] and [2]. That is, if $T(N)$ denotes the maximum expected running time of the algorithm for a collection of $N$ contact surfaces, then we have (recalling that $r$ is assumed to be a constant)

$$
T(N) \leq c_{1} r^{2+\varepsilon} \cdot T\left(\frac{c N}{r} \log r\right)+O\left(N^{2+\varepsilon}\right),
$$

for appropriate constants $c$ and $c_{1}$ (independent of $r$ ). With an appropriate choice of $r$ as a function of $\varepsilon$, it is easily verified that the solution of this recurrence is $T(N)=O\left(N^{2+\varepsilon^{\prime}}\right)$, for any $\varepsilon^{\prime}>\varepsilon>0$, where the constant of proportionality depends on $\varepsilon$ and $\varepsilon^{\prime}$. We have thus shown:

Theorem 2.1. A single connected component of the free configuration space of a $k$ sided polygon, moving in a polygonal region bounded by $n$ edges, can be computed by $a$ randomized algorithm with expected running time $O\left((k n)^{2+\varepsilon}\right)$, for any $\varepsilon>0$, with the constant of proportionality depending on $\varepsilon$.

Remark. The algorithm can be made deterministic, using the deterministic (though rather complicated) construction of $\varepsilon$-nets given by Matoušek [12]. The asymptotic running time of the algorithm remains the same. 
It is interesting to note that our algorithm constructs a representation of the cell $C$ which is suitable for point location. For this we maintain the entire recursive structure that the algorithm computes, in the form of a rooted tree. That is, at the root of that tree we store the decomposition of the cell $C_{0}$ into the subcells $\xi_{\Delta}$, as described above. Each such subcell becomes a child of the root, and stores a similar cell decomposition in the arrangement of the random sample constructed for the corresponding subproblem, and so on. Now, given a query point $q$, we find, by brute force, the subcell $\xi_{\Delta}$ of the top cell $C_{0}$ containing $q$. If no such subcell is found, we conclude that $q$ is not in our cell. Otherwise, we continue the search recursively at the child corresponding to $\xi_{\Delta}$. Thus, we can determine whether $q$ lies in our cell in $O(\log k n)$ time. In terms of the motion-planning application, this means that we can determine in logarithmic time (using $O\left((k n)^{2+\varepsilon}\right)$ preprocessing and storage, for any $\varepsilon>0$ ) whether a given placement of the robot $B$ is free and can be reached from $Z$ via a collision-free path (this is the socalled reachability problem). It is also easy to enhance our data structure with additional information, so that actual motion planning between $Z$ and a query placement can be performed in $O\left((k n)^{2+\varepsilon}\right)$ time.

\section{Acknowledgments}

The authors wish to thank Jirka Matoušek and Leo Guibas for helpful discussions concerning the problem studied in this paper.

\section{References}

1. B. Aronov and M. Sharir, Triangles in space, or building (and analyzing) castles in the air, Combinatorica 10 (1990), 137-173.

2. B. Aronov and M. Sharir, Castles in the air revisited, Discrete Comput. Geom. 12 (1994), 119-150.

3. L. P. Chew and K. Kedem, A convex polygon among polygonal obstacles: placement and high-clearance motion, Comput. Geom. Theory Appl. 3(2) (1993), 59-89.

4. M. de Berg, K. Dobrindt, and O. Schwarzkopf, On lazy randomized incremental construction, Discrete Comput. Geom. 14 (1995), 261-286.

5. L. Guibas and M. Sharir, Combinatorics and algorithms of arrangements, in New Trends in Discrete and Computational Geometry (J. Pach, ed.), Springer-Verlag, New York, 1993, pp. 9-36.

6. D. Halperin, Algorithmic Motion Planning via Arrangements of Curves and of Surfaces, Ph.D. Dissertation, Computer Science Department, Tel Aviv University, July 1992.

7. D. Halperin and M. Sharir, Arrangements and their applications in robotics: recent developments, in The Algorithmic Foundations of Robotics (K. Goldberg, D. Halperin, J.-C. Latombe, and R. Wilson, eds.), A. K. Peters, Boston, MA, 1995, pp. 495-511.

8. D. Halperin and M. Sharir, Almost tight upper bounds for the single cell and zone problems in three dimensions, Discrete Comput. Geom. 14 (1995), 385-410.

9. K. Kedem and M. Sharir, An efficient motion-planning algorithm for a convex polygonal object in twodimensional polygonal space, Discrete Comput. Geom. 5 (1990), 43-75.

10. K. Kedem, M. Sharir, and S. Toledo, On critical orientations in the Kedem-Sharir motion planning algorithm for a convex polygon in the plane, Proc. 5th Canadian Conf. on Computational Geometry, 1993, pp. 204 209.

11. D. Leven and M. Sharir, On the number of critical free contacts of a convex polygonal object moving in 2-D polygonal space, Discrete Comput. Geom. 2 (1987), 255-270. 
12. J. Matoušek, Approximations and optimal geometric divide-and-conquer, Proc. 23rd ACM Symp. on Theory of Computing, 1991, pp. 506-511.

13. J. T. Schwartz and M. Sharir, On the two-dimensional Davenport-Schinzel problem, J. Symbolic Comput. 10 (1990), 371-393.

14. M. Sharir and P. K. Agarwal, Davenport-Schinzel Sequences and Their Geometric Applications, Cambridge University Press, New York, 1995.

15. M. Sharir and S. Toledo, Extremal polygon containment problems, Comput. Geom. Theory Appl. 4 (1994), 99-118.

16. D. D. Sleator, R. E. Tarjan, and W. P. Thurston, Rotation distance, triangulations, and hyperbolic geometry, J. Amer. Math. Soc. 1 (1988), 647-681.

17. R. E. Tarjan, Data Structures and Network Algorithms, SIAM, Philadelphia, PA, 1983.

Received March 2, 1995, and in revised form December 29, 1995. 